\title{
Die Wundgrundzytologie
}

\section{Wound-Bed Cytology}

Bibliografie

DOI $10.1055 / \mathrm{s}-2008-1077474$

Akt Dermatol 2008; 34 :

262-265 @ Georg Thieme

Verlag KG Stuttgart · New York ISSN 0340-2541

Korrespondenzadresse

Dr. med. Pierre Foss

Hautarzt und Wundarzt

Kräwigstraße 8-10

66687 Wadern

pierre.foss@ana-podo.de

\section{Zusammenfassung \\ $\nabla$}

Eine neue Routinediagnostik zur sofortigen zytologischen Beurteilung vor allem chronischer Wunden wird vorgestellt. Sehr hilfreich ist das einfache Verfahren zur schnellen Analyse des bakteriellen Status der Wunde. Mit der Wundgrundzytologie können analog wie bei der gynä-

\section{Einleitung}

Die Beurteilung des bakteriellen Status von Problemwunden ist unbestritten von erheblicher Bedeutung für die Wundheilung. Sie erfolgt bisher vor allem durch bakteriologische Abstriche mit Resistenzprüfungen, den klinischen Aspekt der Wunde und ihrer Umgebung sowie die Werte des Entzündungslabors. Diese Bakteriologie erfordert allerdings mehrere Tage der Auswertung. Eine sofortige, aktuelle Bewertung des bakteriellen Befalls erlauben jedoch zytologische Abstriche des Wundgrundes analog der Vorgehensweise bei gynäkologischen Abstrichen. Es bot sich an, die gynäkologisch-zytologischen Abstrichmethoden auch zu Analysen des Wundgrundes zu nutzen $[1,2]$. Sie ist eine wichtige, unverzichtbare Bewertungsstütze und Hilfe in der antibakteriellen Behandlung dieser Problemwunden geworden. Diese Erstveröffentlichung der Wundzytologie soll dazu dienen, diese einfache, aber sehr aussagekräftige Zytologiediagnostik einem weiten Kreis der Kollegen bekannt zu machen.

\section{Methode \\ $\nabla$}

Die Wunde sollte vor der Probennahme gesäubert werden. Für den Abstrich haben sich Rundbürsten bewährt, die im medizinischen Fachhandel zu beziehen sind (Celltip Abstrichbürsten, kologischen Abstrichzytologie alle üblichen und speziellen Färbetechniken genutzt werden. Zur schnellen Übersicht im Präparat hat sich die Methylenblau-Färbung bestens bewährt. Das zytologische Verfahren ist eine wertvolle Ergänzung zur Wundhistologie und zur bakteriologischen Abstrichdiagnostik.

1 Packung enthält 10 Beutel zu je 10 Bürsten. Bezug über Laborbedarf). Die Bürsten können nicht über den Sprechstundenbedarf abgerechnet werden. Diese weichen Zytologiebürsten werden in der Gynäkologie in verschiedenen Formvarianten verwendet. Die hier gezeigte, fast walzenartige Bürstenform ist die geeignete Variante für die Wundgrundzytologie. Die Bürste ist möglichst tangential und unter leichtem Andruck und Drehung über die Wunde bzw. den Wundgrund zu führen. Es sollte darauf geachtet werden, dass nicht zuviel Fibrin- bzw. Belagschleim abgehoben wird, damit der nachfolgende Ausstrich auf dem Objektträger nicht zu dick wird und damit schlecht durchlichtmikroskopisch zu inspizieren ist ( Abb. 1, 2).

Der Objektträger wird nun mit Methylenblau dünn überschichtet ( $\bullet$ Abb.3), dann ein Deckglas aufgesetzt und der Rest des Methylens an den Objektträgerseiten abgetupft. Danach wird Immersionsöl auf das Deckglas getropft und der Ausstrich kann mikroskopiert werden. Es besteht auch die Möglichkeit den Ausstrich mit der Flamme in Hitze zu fixieren. Die gesamte Präparation erfordert weniger Zeit als 1 Minute und ein einfaches Durchlichtmikroskop mit einer 40×-, 62×und 100×-Objektivvergrößerung ( Abb. 4-6).

Die dargebotenen mikroskopischen Bilder der Wundgrundzytologie wurden mit dem Spezialmikroskop DM 4000 der Fa. Leica, Wetzlar digital fotografiert und digital nachbearbeitet. Die Aus- 


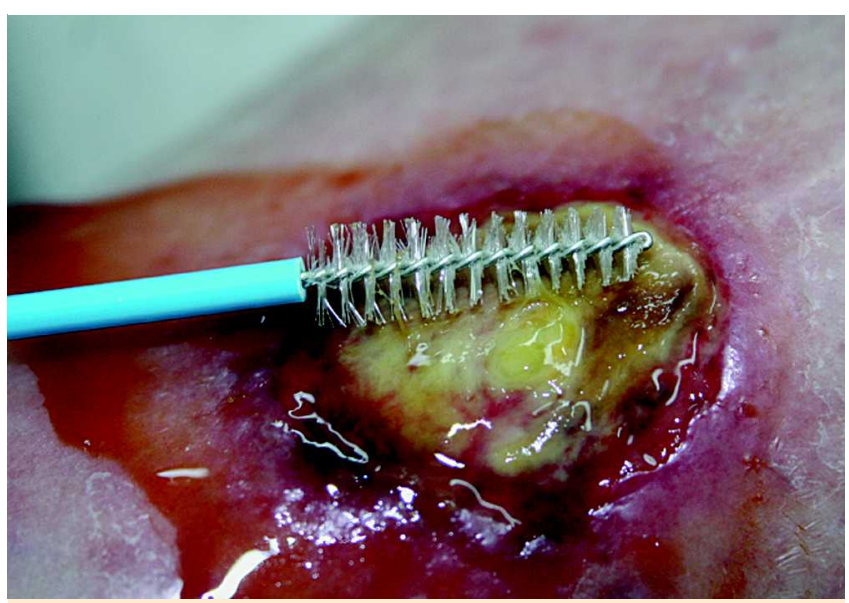

Abb. 1 Die weiche Abstrichbürste wird unter leichtem Andruck und Rotation über den Wundgrund geführt. Es können schichtweise Abnahmen erfolgen bis in die Tiefe des Wundgrundes. Die Abnahme von zuviel Fibrinmaterial ist zu vermeiden, damit der Ausstrich auf dem Objektträger nicht zu dick wird.

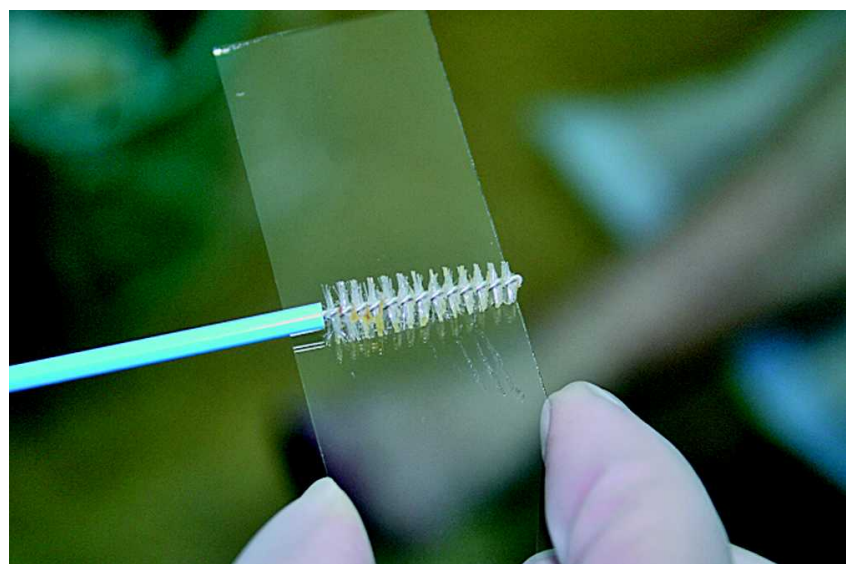

Abb. 2 Die Abstrichbürste wird unter leichtem Andruck und Drehung über den Objektträger geführt.

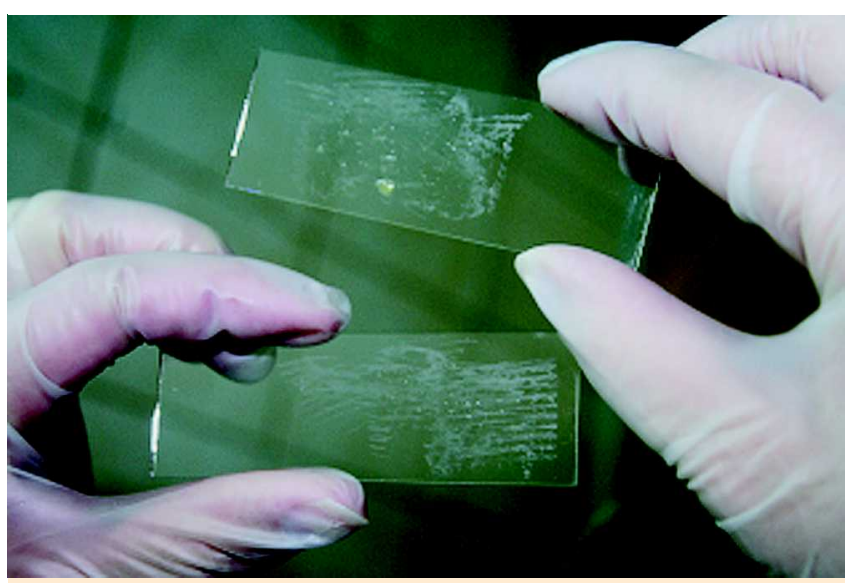

Abb. 3 Der bestrichene Objektträger wird mit Methylenblau-Lösung überschichtet. Dieser wird dann mit einem länglichen Deckglas bedeckt und kann dann wie üblich mikroskopiert werden. striche wurden mit Methylblau gefärbt. Die Bilder mit dem 100×-Objektiv wurden unter Öl-Immersion angefertigt.

\section{Aussagemöglichkeiten}

$\nabla$

Mit der einfachen und universellen Methylenfärbung lassen sich sehr gut Bakterien, Zellen, Zellkerne und Fibrinfäden anfärben. Die Bakterien können gut mit dem 62×- oder100×-Objektiv in der Anzahl und der Lage zu den Zellen und zum Fibrin dargestellt und in der Menge eingeschätzt werden. Die Fibrinfäden immobilisieren die Bakterien und hemmen so deren Fortbewegung und Teilung. Liegen viele Bakterien außerhalb der Fibrinfäden und Zellen, vor allem in größeren Kolonien, so ist von einer behandlungsbedürftigen Wundinfektion auszugehen. Es können schonend aus einer Wunde mehrere Abstriche durchgeführt werden. Dies kann durchaus sinnvoll sein, um die Beläge an verschiedenen Stellen und in unterschiedlichen Tiefen zu analysieren und so einen validen und relevanten Gesamtüberblick zu gewinnen ( $\bullet$ Abb. 7). Der Wundgrund kann mit dem scharfen Löffel oder der Ringkürette aufgeraut werden, um damit noch tieferes Wundmaterial gewinnen zu können.

\section{Fehlerquellen \\ $\nabla$}

Der unerfahrene Mikroskopiker kann verschiedene Strukturen, Artefakte und Schrumpfungsphänomene als Sporen und Bakterien fehlinterpretieren. Im Wundgrund sind kleinste Serumtropfen, Lymphtröpfchen mit Eiweißpartikel und je nach Wundtherapie auch Partikel der externen Auflagen vorhanden. So finden sich Zellstoffreste, Kompressenfasern und teils auch Reste von Fettgazen in der Wunde, die nicht als Hyphenfäden und Bakterien eingestuft werden sollten. Die Hyphen zeigen regelmäßige klare Septen, die sich bei den anderen fasrigen Strukturen nicht darstellen. Vor allem wenn Hydrokolloide oder Hydrogele genutzt werden, sollte vor der zytologischen Abnahme die Wunde gesäubert oder abgespült werden, da kleinste Hydrogelkugeln leicht mit großen Sporen verwechselt werden können. Wenn dickere Beläge vorhanden sind, so sollten diese mindestens teilweise abgetragen oder abgehoben werden und mit der Zytologiebürste das Wundmaterial in der Tiefe der Wunde durch Drehen der Bürsten entnommen werden.

\section{Abrechnungsmodalitåten \\ $\nabla$}

GKV/EBM: pro Abstrich: 32045, 32050 (bei Gram-Färbung), 10340 ( wenn tiefere Entnahmen erfolgen ).

PKV/GOÄ: pro Abstrich: 297, 3509, evtl. 3510, 2401 (bei tieferen Entnahmen), 4852 (Punktate).

\section{Diskussion}

$\nabla$

Mit dieser einfachen Methode lassen sich schnell und sofort aussagekräftige zytologische Bewertungen des Wundgrundes durchführen. Der besondere Wert dieser Methode liegt in der Möglichkeit, eine aktuelle Beurteilung des bakteriellen Status der Wunde durchführen zu können. Die Wundgrundzytologie ist somit eine ideale Ergänzung zur Abstrichbakteriologie, zur Histologie und zur klinischen Inspektion der Wunde. Alle übli- 


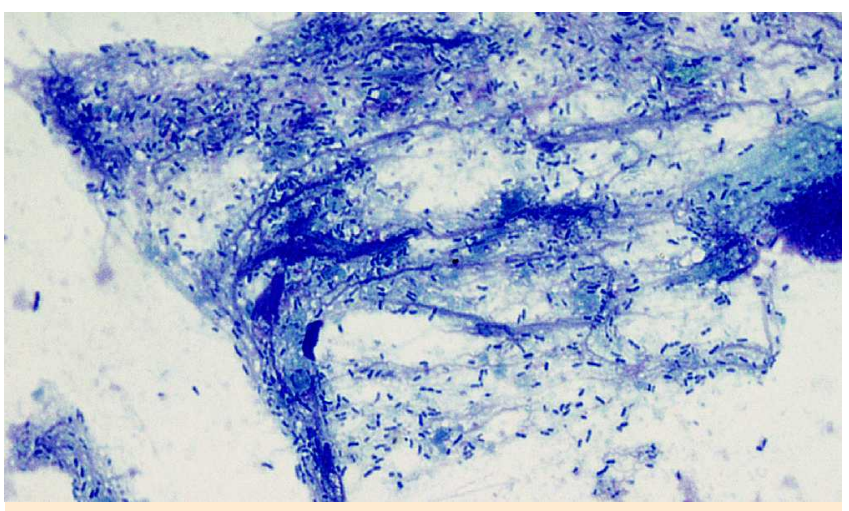

Abb. 4 62×-Objektiv: Im Fibrinbelag sind deutlich Bakterien zu erkennen. Auch außerhalb der Beläge sind Bakterien gut zu sehen. Dieser Befund ist pathologisch. Es handelt sich um eine massive bakterielle Infektion der Wunde.

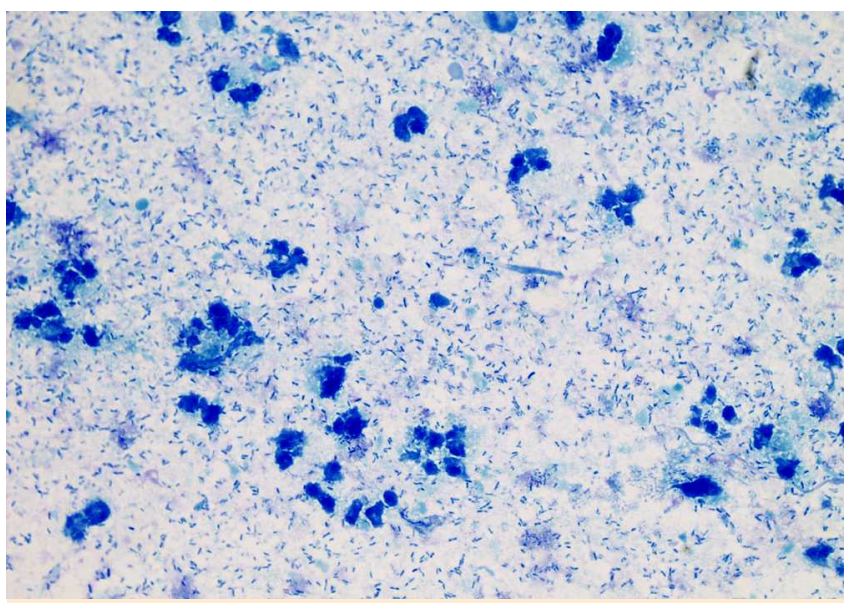

Abb. 5 62×-Objektiv: Außerhalb der Fibrinbeläge und Zellen sind massive Bakterienansammlungen zu erkennen. Der Befund ist hoch pathologisch.

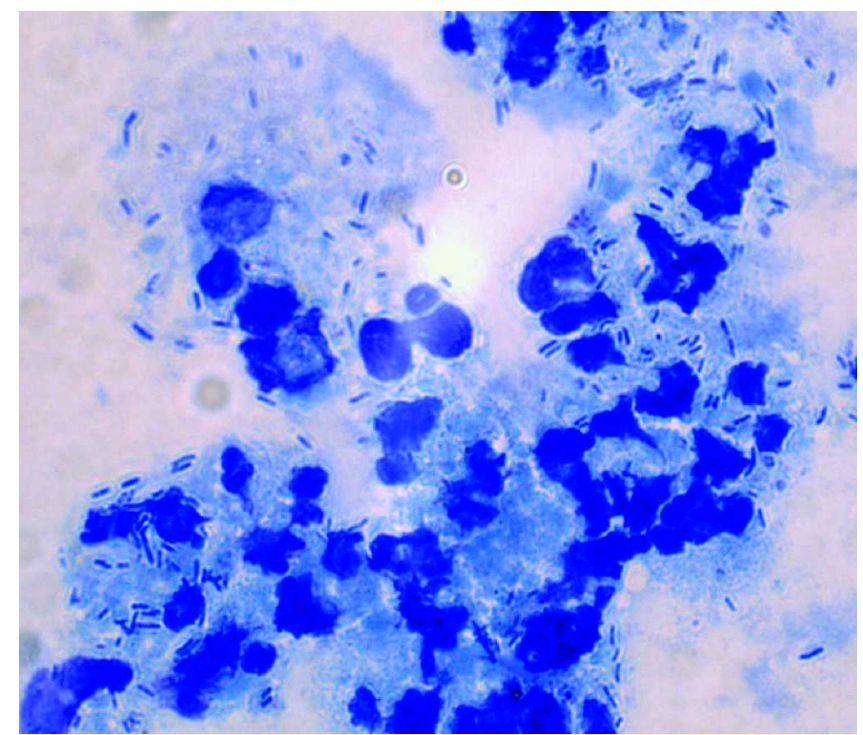

Abb. 6 100×-Objektiv: Die Zellen mit ihren teils zerquetschten Zellkernen sind gut zu erkennen. Viele Bakterien liegen in den Zellen und außerhalb der Zellen. Der Befund ist deutlich pathologisch. chen zytologischen Färbetechniken können hierbei auch genutzt werden. Falls zweckmäßig können Fixiersprays und Objektträger mit speziellen Haftbeschichtungen verwendet werden.

Die Analysen der Epithelzellen können teils analog den Grundsätzen der gynäkologischen Zytologie und speziell der gynäkologisch-zytologischen Krebsdiagnostik bewertet werden [1,2].

Mit einiger Übung lässt sich leicht - zusammen mit dem klinischen Befund - eine valide Einschätzung des bakteriellen Status (Kontamination, Kolonisation, Infektion) gewinnen. Die Möglichkeit, eine Wundgrundzytologie mehrfach in der Fläche und Tiefe zu machen, garantiert einen wirklichen Überblick über den bakteriellen Zustand der Wunde. In der Tiefe des Wundgrundes können vor allem Anaerobier detektiert werden. Störende Wundbeläge werden in meiner Praxis sehr schonend mit dem Erbium-YAG-Laser abgetragen $(30 \mathrm{~mJ} / 10 \mathrm{~Hz}$, Spotdurchmesser 2-3 mm) (๑ Abb. 7).

Generell sollte bei allen Wunden, die nicht rasch abheilen und eine Entzündung sowie Schmerzen zeigen, an eine tiefere bakterielle Gewebeinfektion mit Problemkeimen gedacht werden, die evtl. einer resistenzgeprüften, gezielten, oralen Antibiose bedürfen. Vor allem dürfen Rötungen des Unterschenkels nicht nur als kontaktallergische Hautveränderungen angesehen werden. Wenn erhebliche Schwellungen des Unterschenkels bestehen, mit Schmerzen und ohne Juckreiz, muss an ein Erysipel gedacht werden. Das kontaktallergische Erythem juckt meist erheblich, zeigt daher oft Kratzspuren und verursacht keine Schmerzen. Wenn eine Kolonisation bei schlechten Durchblutungsverhältnissen (pAVK) oder Neigung zu Erysipelen, Diabetes usw. vorliegt, sollte eine suffiziente lokale Desinfektion mit Präparaten erfolgen, die eine Desinfektion der Wunde über mehrere Tagen erlauben (Prontosan Wound $\mathrm{Gel}^{\circledR}$ ). Falls dies nicht möglich sein sollte, ist eine tägliche Inspektion der Wunde bis zum Eindämmen der Infektion notwendig. Hierzu können dann auch andere übliche und empfohlene Wunddesinfektionsmittel genutzt werden. Die bakterielle Sanierung der Wunde lässt sich nachfolgend bei jedem Verbandswechsel schnell mit der Wundgrundzytologie überprüfen. Die Wundgrundzytologie sollte nach Ansicht des

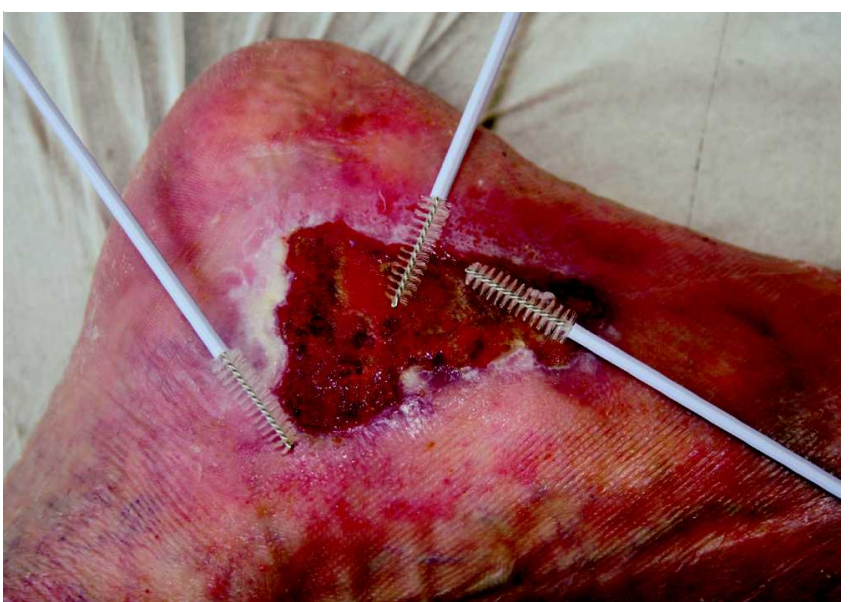

Abb. 7 Nach der Abtragung mit Erbium-YAG-Laser: Wundgrundzytologie von den 3 Arealen. Siehe Position der Bürsten. Zytologisch zeigte sich ein sehr variabler Bakterienbefall. In dem mittleren Areal wurden große fischzugartige Bakterienkolonien gesehen, in den anderen Arealen locker verteilte und fibringebundene Bakterien. Der Bakterienstatus und das klinische Bild rechtfertigen eine orale Antibiose nach Kenntnis der Bakteriologie mit Resistenzprüfung. 
Autors eine Routineuntersuchung chronischer Wunden werden. Sie ist schnell, schonend und sehr kostengünstig durchführbar und erlaubt Varianten und zusätzliche Analysen auch des Zellbesatzes in der Wunde.

Gemäß den Richtlinien und Erkenntnissen der gynäkologischen Krebsdiagnostik (Kern-Plasma-Relation, Kernform, Zellformen usw.) sind hiermit auch Aussagen über die Dignität der Wundzellen möglich [1,2]. Diese Veröffentlichung hat jedoch bewusst den Fokus auf die wesentlich häufigere bakteriologische Diagnostik gelegt.

Wünschenswert wären zukünftig zytologische Marker für Problembakterien, die eine sofortige Überprüfung und Erkennung weniger, allerdings wichtiger Keime im zytologischen Präparat ermöglichen würden. Dies könnte mithilfe von Fluoreszenz-Antikörpern geschehen, die auf den Objektträger aufgetragen, die Problemkeime sofort unter dem Mikroskop sichtbar machen würden.

Ein weiterer Gesichtspunkt sollte hier noch angesprochen werden:

Die bakterielle Kulturdiagnostik soll zwar deutlich zuverlässiger sein als die mykologische Kulturdiagnostik, weil die bakteriellen Erreger sich gut anzüchten lassen, doch gelingt oft die Anzucht der Bakterien nicht, wenn diese vorher antibiotisch oder mit Haut- und Wunddesinfektionsmitteln vorbehandelt wurden. Diese therapeutischen Bedingungen liegen aber oft bei chronischen Wunden vor. Die Wundgrundzytologie hat diesen Nachteil nicht und könnte so zur Klärung falsch negativer Kulturbefunde dienen (Kosten bei GKV-Patienten).

Hinzukommt, dass die Kosten der bakteriellen Kulturdiagnostik nicht unerheblich sind. Eine MRSA-Befundung kostet etwa 19 Euro bei negativem Ergbenis, ein positiver Befund etwa 29 Euro durch die Nachbearbeitung. Die Pseudomonas-aeruginosa-Diagnostik kann Kosten von 29 Euro plus 15 Euro Nachbereitung verursachen.

Die Wundgrundzytologie könnte als Pfadfinder und Absicherung für die bakterielle Kulturdiagnostik dienen. Aus dem Wundareal sollten an verschiedenen Stellen zytologische Abstri- che entnommen werden und aus den Bereichen bzw. dem Bereich, der die meisten Bakterien enthält, wird dann ein aussagekräftiger Abstrich entnommen. Dem Mikrobiologen könnte der positive zytologische Befund und evtl. die relevante interne bzw. externe Therapie mitgeteilt werden, damit dieser Kollege den bakteriellen Kulturbefund besser zu interpretieren vermag. Eine erste Vorstellung der Wundgrundzytologie erfolgte von mir in der Fachtagung „Dermatologische Praxis 2008“ in Frankenthal, am 4. April 2008, während meines Vortrages „Aktuelle Aspekte der Wundversorgung“.

Anmerkung: Die Praxis des Autors ist spezialisiert für die Behandlung chronischer Wunden. Der Autor führt die Wundgrundzytologie seit 2 Jahren routinemäßig durch.

\section{Abstract}

\section{Wound-Bed Cytology}

A new routine diagnostic method for immediate cytological assessment of wounds, primarily chronic ones, is presented. The simple method is very helpful for quick analyses of the bacterial status of wounds. For wound bed cytology, all standard and special staining techniques may be used, making it analogous to gynecological smear cytology. Methylene blue staining is an excellent method for a quick overview of a specimen. The cytological method is a valuable addition to wound histology and bacterial smear diagnostic procedures.

\section{Literatur}

1 Jacques J, Alan BPN. Gynäkozytologie und Krebsvorsorge in der gynäkologischen Praxis. Bern: Hans Huber, 1993

2 Soost HJ, Baur S. Gynäkologische Zytodiagnostik. Stuttgart: Thieme, 1990 